\title{
Evidence for Transmission of Taenia solium Taeniasis/Cysticercosis in a Rural Area of Northern Rwanda
}

\section{OPEN ACCESS}

Edited by:

Rodrigo Morchón García,

University of Salamanca, Spain

Reviewed by:

Elizabeth Ferrer

University of Carabobo, Venezuela

Maria J. Perteguer,

Instituto de Salud Carlos III

(ISCIII), Spain

*Correspondence:

Fernando Jorge Bornay-Llinares

f.bornay@umh.es

${ }^{\dagger}$ These authors have contributed equally to this work and share senior authorship

Specialty section:

This article was submitted to

Parasitology,

a section of the journal

Frontiers in Veterinary Science

Received: 22 December 2020

Accepted: 23 February 2021

Published: 20 April 2021

Citation:

Acosta Soto L, Parker LA Irisarri-Gutiérrez MJ, Bustos JA, Castillo Y, Perez E, Muñoz-Antoli C,

Esteban JG, García HH and Bornay-Llinares FJ (2021) Evidence

for Transmission of Taenia solium Taeniasis/Cysticercosis in a Rural Area of Northern Rwanda.

Front. Vet. Sci. 8:645076. doi: 10.3389/fvets.2021.645076

\author{
Lucrecia Acosta Soto ${ }^{1}$, Lucy Anne Parker ${ }^{2,3}$, María José Irisarri-Gutiérrez ${ }^{4,5}$, \\ Javier Arturo Bustos ${ }^{6,7}$, Yesenia Castillo ${ }^{7}$, Erika Perez ${ }^{6}$, Carla Muñoz-Antoli ${ }^{4}$, \\ José Guillermo Esteban ${ }^{4}$, Héctor Hugo García ${ }^{6,7 \dagger}$ and Fernando Jorge Bornay-Llinares ${ }^{1 * t}$ \\ ${ }^{1}$ Área de Parasitología del Departamento de Agroquímica y Medioambiente, Universidad Miguel Hernández de Elche, \\ Alicante, Spain, ${ }^{2}$ Departamento de Salud Pública Historia de la Ciencia y Ginecología, Universidad Miguel Hernández de \\ Elche, Alicante, Spain, ${ }^{3}$ Centro de Investigación Biomédica en Red de Epidemiología y Salud Pública (CIBERESP), Madrid, \\ Spain, ${ }^{4}$ Área de Parasitología, Dpto. Farmacia y Tecnología Farmacéutica y Parasitología, Facultat de Farmàcia, Universitat \\ de València, Valencia, Spain, ${ }^{5}$ Dpto. de Ciencias de la Salud, Facultad de Ciencias Biomédicas, Universidad Europea de \\ Madrid, Madrid, Spain, ${ }^{6}$ Cysticercosis Unit, Instituto Nacional de Ciencias Neurológicas, Lima, Peru, ${ }^{7}$ Center for Global \\ Health, Universidad Peruana Cayetano Heredia, Lima, Peru
}

Cysticercosis is a parasitic infection caused by the metacestode larval stage (cysticercus) of Taenia solium. In humans, cysticercosis may infect the central nervous system and cause neurocysticercosis, which is responsible for over 50,000 deaths per year worldwide and is the major cause of preventable epilepsy cases, especially in low-income countries. Cysticercosis infection is endemic in many less developed countries where poor hygiene conditions and free-range pig management favor their transmission. A cross-sectional study was conducted in 680 children from a rural primary school in Gakenke district (Northern province of Rwanda). Stool samples were collected from participants and analyzed using the Kato-Katz method (KK), formol-ether concentration (FEC), and/or copro-antigen enzyme-linked immunosorbent assay (CoAg-ELISA) to detect taeniasis. Blood samples were collected and analyzed using enzyme-linked immunoelectrotransfer blot (EITB) and antigen enzyme-linked immunosorbent assay (Ag-ELISA) to detect human cysticercosis. The overall proportion of taeniasis positivity was $0.3 \%$ (2/680), and both cases were also confirmed by CoAg-ELISA. A total of $13.3 \%$ (76/572) of the children studied were positive to cysticercosis (T. solium-specific serum antibodies detected by EITB), of whom 38.0\% (27/71) had viable cysticercus (T. solium antigens by Ag-ELISA). This study provides evidence of the highest cysticercosis prevalence reported in Rwanda in children to date. Systematic investigations into porcine and human cysticercosis as well as health education and hygiene measures for $T$. solium control are needed in Gakenke district.

Keywords: Taenia solium, taeniasis, cystcercosis, children, Gakenke, Rwanda 


\section{INTRODUCTION}

In Taenia solium parasitic infections, humans are the only natural definitive host (taeniasis) while pigs are the intermediate hosts of larval stage (swine cysticercosis) (1). Human cysticercosis results when people become intermediate hosts after ingestion of microscopic viable eggs via the fecal-oral route from T. solium tapeworm carriers (2). The embryo is released (oncosphere), and it traverses the intestinal mucosa after ingestion. Later, it is transported by the circulatory system and dispersed by the organism producing cysts (cysticerci). The most common locations of cysts are the striated muscle, eyes, or heart tissue and central nervous system $(3,4)$. The clinical manifestations of cysticercosis are dependent on the number and location of cysticerci within the body (4). Some individuals with cysticercosis will exhibit or develop no symptoms (asymptomatic) or very mild symptoms. Many individuals with cysticercosis have central nervous system involvement (neurocysticercosis) resulting in headache, epileptic seizures, blindness, mental disturbance, and even death $(3,5)$. Neurocisticercosis (NCC) is the most common parasitic disease in the human nervous system and the most common cause of epilepsy in low-income countries (6).

Currently, cysticercosis is one of the 17 major Neglected Tropical Diseases (NTDs) identified by the WHO as a focus for research and control (7). It is widely prevalent where humans and domestic pig raising coexist. In many developing countries in Central and South America, Africa, and Asia, cysticercosis has major public health implications in humans and pigs $(5,8-$ 11). It is in these places where poverty, poor education, lack of access to diagnosis, and limited management capacity, together with the absence of appropriate prevention measures and control strategies, make it highly endemic (11-13).

The distribution of T. solium taeniosis/cysticercosis in Africa is unclear but porcine and human cysticercosis are considered (hyper)-endemic in Central Africa (Rwanda, Burundi, the Democratic Republic of Congo, and Cameroon) $(14,15)$. In the last 20 years, pig production has increased significantly in the Eastern and Southern Africa (ESA) region, especially in rural, resource-poor, smallholder communities $(11,14)$. Several studies show a high prevalence of porcine cysticercosis in countries bordering Rwanda. In Uganda, prevalences ranging from 7.1 to $45 \%$ have been observed in urban areas in contrast to low percentages of $0.12-10.8 \%$ found in rural areas with an observed increment in recent years $(9,16-18)$. A prevalence of $41.2 \%$ has recently been reported in the Democratic Republic of Congo, where the overall prevalence of pigs with active cysticercosis did not significantly differ between the market and the village study sites but was much higher than previously observed by Chartier et al., in $1990(19,20)$. There are no current data for Burundi, but prevalence ranges from 2 to $39 \%$ were observed 20 years ago (21). In Tanzania, farm prevalence of porcine cysticercosis was between 17.4 and $18.2 \%$ in lingual examination or slaughterslab prevalence by routine meat inspection respectively, while a maximum of $33.3 \%$ has been reported by cysticercal antigens by ELISA (Ag-ELISA) (22-29).

In contrast, Taeniasis has been poorly studied in humans from these countries (30). Eggs of Taenia sp. in feces have been reported in Uganda (31). Taeniasis prevalence ranges between 0 and $1.0 \%$ have been observed in schoolchildren of Burundi (21). However, prevalence of taeniasis ranging from 0.4 to $5.2 \%$ by the Kato-Katz technique or $2.3-5.2 \%$ by copro-Ag-ELISA has been estimated in Tanzania $(32,33)$.

Regarding human cysticercosis, one study estimated $21.6 \%$ prevalence of circulating antigen in the Democratic Republic of Congo (34). Several studies in human cysticercosis reveal the strong association between neurocysticercosis and epilepsy in these countries $(35,36)$. In Burundi, cysticercosis has been observed in $4.9-31.5 \%$ of epileptic patients, compared to $4.2 \%$ in controls $(21,37-39)$. On the other hand, a seroprevalence of up to $11.7 \%$ has been observed in epileptic patients and in $2.8 \%$ of controls (21). Cysticercosis was the cause of seizures in $25 \%$ of epileptic patients $(38,40)$. In Tanzania, cysticercosis prevalence of $16-17 \%$ was estimated and it was demonstrated that NCC contributed significantly to epilepsy in adults (32, 41-43). Given these data, it is likely that the situation in Rwanda is similar.

Rwanda has long since been known as a hyperendemic country for Taeniasis/cysticercosis. However, there are few research studies carried out in this country. Already in 1959, $20 \%$ of pigs were found to be infected with cysticercosis (44). From 2000 to 2011, pork production in Rwanda increased by $7.8 \%$ (45); however, an overall swine cysticercosis prevalence of $3.9 \%$ was found in farms, $9.2 \%$ in markets, and $4 \%$ in butchers (46). In 1956, the first case of human cysticercosis was reported in Rwanda (47). Since then, initial reports have reported isolated cases of disseminated ocular (48) and cutaneous cysticercosis (49, 50). In 1964, the presence of eggs of Taenia spp. in populations of the Batwa and Hutu tribes in the Northern and Southern regions of the country was detected (51). Later, T. solium cysticerci was detected in $7 \%$ of 300 autopsies in Butare (52). In Kigali and Butare, 21 and $21.8 \%$ of people with epilepsy are estimated to be seropositive for cysticercosis, respectively $(53,54)$. To date, there have been no studies in children.

During a coproparasitological study in the school population of Northern Rwanda, eggs from tapeworms were detected in two schoolchildren. After collecting the strobila, the morphology of mature proglottids allowed us to identify the species as T. solium in both cases. This observation motivated us to investigate the presence of cysticercosis in the school population of Nemba, with the prospect of introducing control activities.

\section{METHODS}

\section{Study Area}

Rwanda is a small landlocked country in Central Africa, bordering Uganda to the North, Burundi to the South, Democratic Republic of the Congo to the West, and Tanzania to the East. The estimated population in 2012 was 10.4 million people, thus supporting the densest population in continental Africa, with most of the population engaged in subsistence agriculture (55). A verdant country of fertile and hilly terrain with altitudes varying from $950 \mathrm{~m}$ to $4,519 \mathrm{~m}$, the small republic bears the title "Land of a Thousand Hills." The District of Gakenke is one of the five districts of the Northern Province of Rwanda. This 


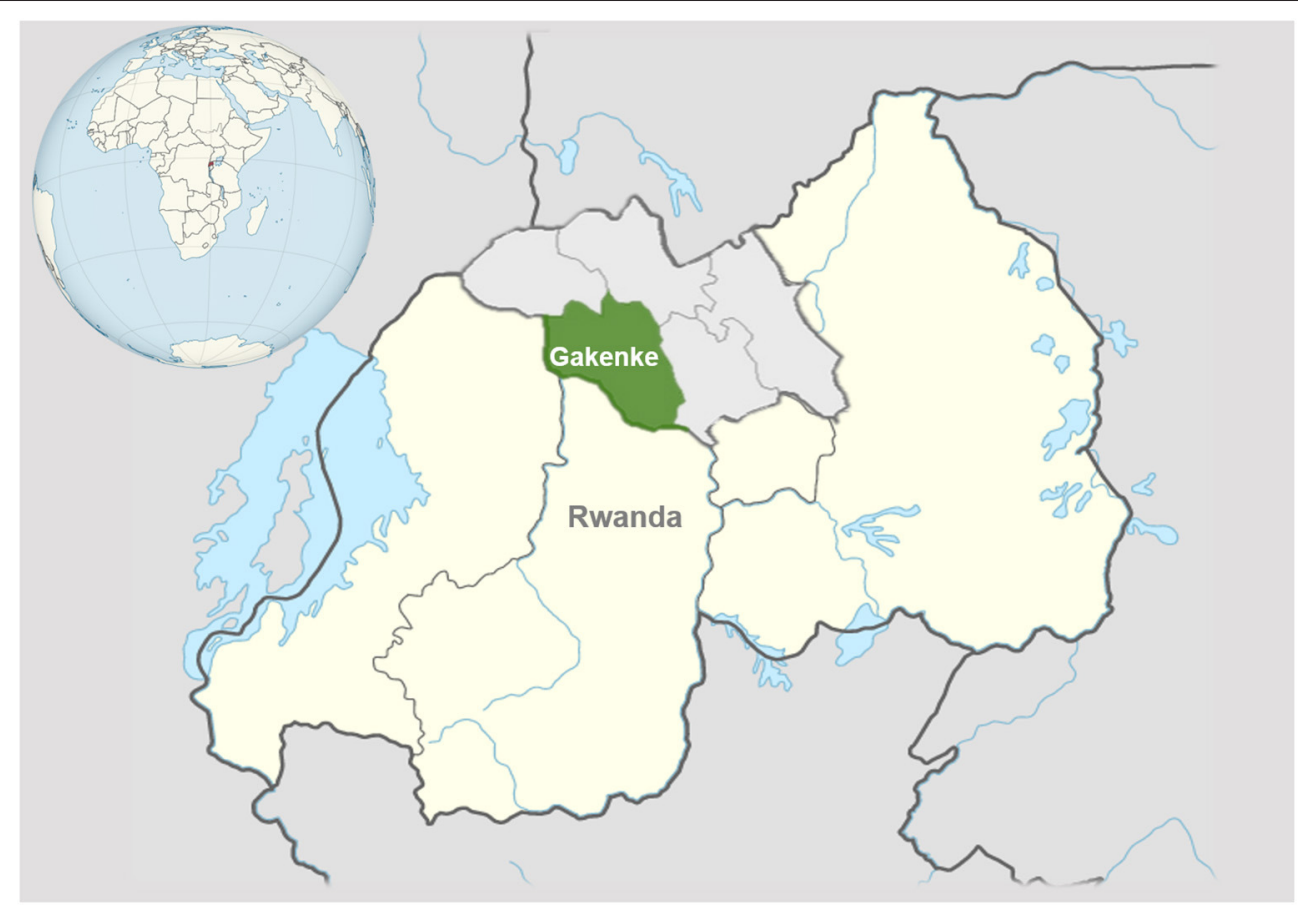

FIGURE 1 | Study location map. District of Gakenke, Northern Province, Rwanda.

district is divided into 19 administrative sectors made of 97 cells, 617 villages, and 345,487 inhabitants living in a total area of 104 $\mathrm{km}^{2}$ with a population density of $473 / \mathrm{km}^{2}$ (56). Figure 1 shows a schematic map of the study area.

Gakenke district is characterized, in general, by high inclined hills separated by rivers and marshlands. The climate in Gakenke district is generally the type of humid climate with the average annual temperature varying between 16.0 and $29.0^{\circ} \mathrm{C}$. The rainfalls are relatively abundant with a scale between 1,100 and $1,500 \mathrm{~mm}$ per year (56).

\section{Population}

Between July and September 2011, the Parasitology Area of University Miguel Hernández de Elche (UMH; Spain) conducted a cross-sectional study in Nemba school I, Gakenke district of Rwanda, as part of an initial phase of a school health program. A total of 771 schoolchildren (371 girls and 400 boys) attended primary education (2nd to 6th grade) and were eligible for inclusion in the study. A total of 708 students (357 boys and 351 girls aged between 6 and 18 years of age; mean $\pm \mathrm{SD}=11.00$ \pm 2.33 years) were eligible for the study after their parents or guardians provided informed consent. A total of 680 answered the questionnaire and provided stool sample, while 572 provided blood sample (see Figure 2).

\section{Sampling and Parasitological Tests}

After collection, fresh feces samples were processed and quantified in situ (UMH headquarters in Nemba, Rwanda) by the method described by Kato-Katz following WHO recommendations (57). A 41.7-mg sample of fresh feces was used as template according to the Helm teste ${ }^{\circledR}$ manufacturer's recommendations (Bio-Manguinhos/Fiocruz, Brazil). If taeniid eggs were observed, $2 \mathrm{~g}$ of Niclosamide and $1 \mathrm{ml}$ of Duphalac ${ }^{\circledR}$ oral solution ( $667 \mathrm{mg} / \mathrm{ml}$ of lactulose) was administered as a laxative to recover the adult where possible.

Additionally, part of each stool sample was preserved in $10 \%$ buffered formalin (1:3) (40-ml Falcon tube) and another part was preserved in ethanol $70 \%$ (2-ml tube). Sera samples were obtained from whole blood collected of every child by centrifugation and stored at $4^{\circ} \mathrm{C}$ in Nemba's Hospital until sent to $\mathrm{UMH}$, Spain. Later, all samples were sent to $\mathrm{UMH}$ and stored at $-20^{\circ} \mathrm{C}$ until processing. Formalin-embedded samples were sent to the Department of Parasitology of University of Valencia (Spain) where a formol-ether concentration was realized (58). Finally, tree drops of sediment with Lugol's iodine solution were analyzed under a microscope. Sera and fecal samples preserved in ethanol $70 \%$ were subsequently sent to the diagnostic laboratory of the Center for Global Health of the Universidad Peruana Cayetano Heredia (UPCH, Lima, Peru) for processing by T. solium-specific tests [Coproantigen ELISA, serum antigen detection (Ag-ELISA), and serum antibody detection by enzymelinked immunoelectrotransfer blot (EITB) assay using lentil lectin-purified parasitic glycoprotein antigens].

\section{Taeniasis: Coproantigen Detection by ELISA (CoAg-ELISA)}

In an attempt to find additional carriers of adult $T$. solium worms, a subgroup of 144 fecal samples ( $21 \%$ of the total) were also screened by coproantigen $\operatorname{ELISA}(59,60)$. Aliquots 


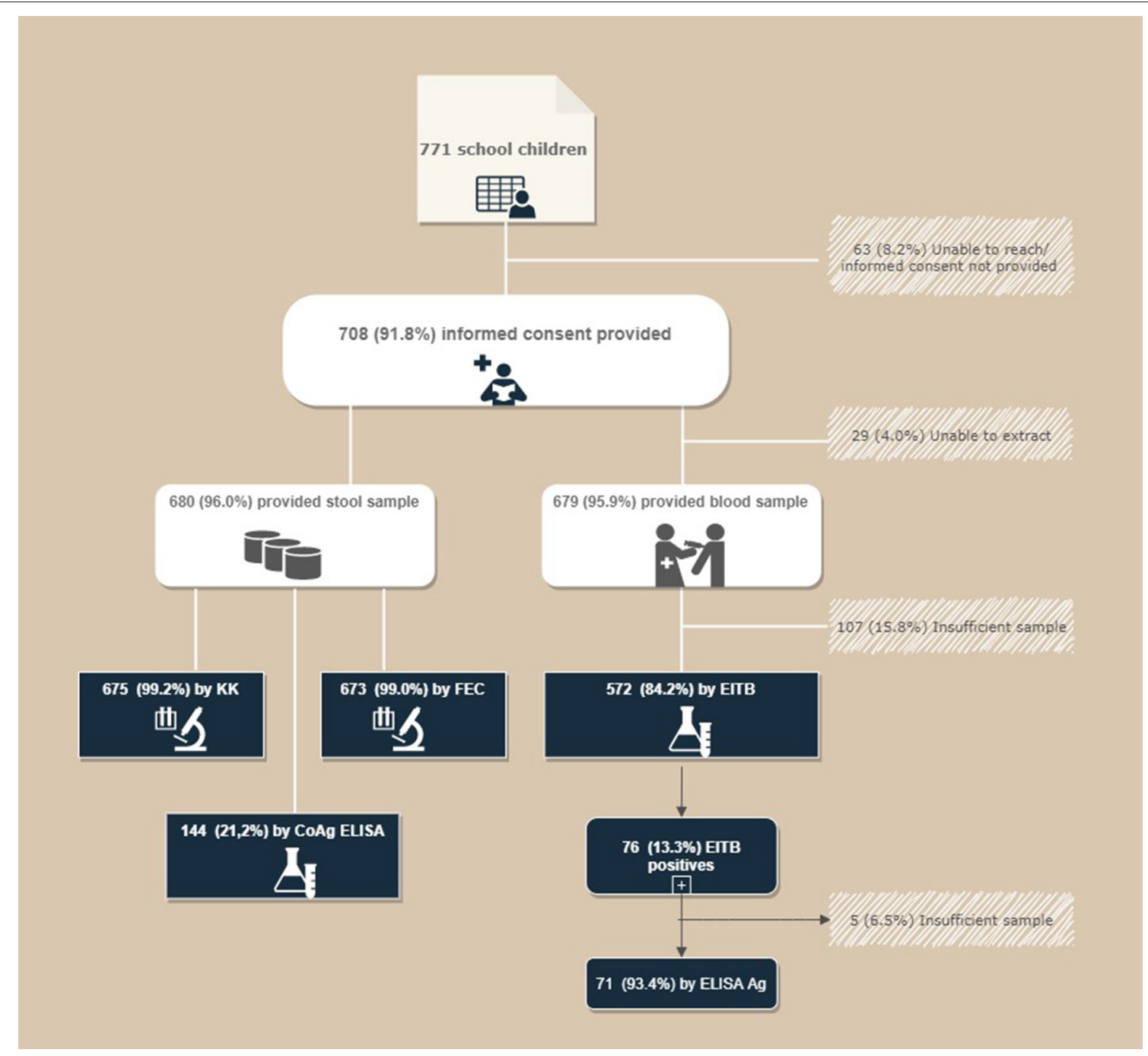

FIGURE 2 | Flow chart for the recruitment of patients.

$(1.5 \mathrm{ml})$ of the stool supernatant were used for CoAg-ELISA after centrifugation at room temperature at 3,200 $\mathrm{g}$ for $10 \mathrm{~min}$. The CoAg-ELISA technique was performed as described by Allan et al., by using hyperimmune rabbit anti-T. solium IgG as the capture antibody and peroxidase-labeled goat anti- $T$. solium IgG as a conjugate. Processed samples were read with a spectrophotometer (Molecular Devices Inc., Sunnyvale, CA) at $650 \mathrm{~nm}$. Using a known positive pool (P1), we calculated the percentage of positivity (PP) as [optical density (OD) of the sample]/(OD of P1) 100, in order to increase the comparability of the results between plates. A cutoff was determined using a receiver operating characteristic (ROC) curve (61).

\section{Cysticercosis-Specific Tests}

\section{Anti-T. solium Antibody Detection}

A total of 572 sera samples were processed by EITB. The methodology used to perform the test was the same described by Tsang et al. (62) and González et al. (63). An immunoblot of seven cysticercus glycoproteins (GP50, GP42-39, GP24, GP21, GP18, GP14, and GP13), purified by lentil lectin-purified chromatography, gives close to $100 \%$ specificity and a sensitivity varying from 70 to $90 \%$ (62). This EITB has been widely used for the diagnosis of cysticercosis in human and pig serum samples (64).

\section{T. solium Antigen Detection by ELISA}

In order to demonstrate the presence of active infections, an anti-T. solium antibody-positive subgroup of children (71 EITB positive samples) were processed in quest by ELISA, to detect circulating cysticercal antigen in the serum $(65,66)$. A mAb-based ELISA for the detection of circulating antigens was used to detect circulating parasite antigen as described by Brandt et al. in 1992 (67) and later adapted by Van Kerckhoven et al. and Dorny et al. $(68,69)$. The assay uses Nunc MaxiSorp plates sensitized with a trapping mAb (B158C11A10) in bicarbonate buffer at $5 \mu \mathrm{g} / \mathrm{ml}$. After blocking, serum samples 
(pretreated with 5\% trichloroacetic acid to break existing immune complexes) are added, followed by the second mAb (B60H8A4-BIOT), streptavidin, o-phenylenediamine (OPD/H2O2) as substrate/chromogen, and incubated in the dark for $15 \mathrm{~min}$. The reaction is stopped with $\mathrm{H}_{2} \mathrm{SO}_{4}$ and plates are read at $490 / 650 \mathrm{~nm}$. To minimize inter-plate variation, we used antigen ratio instead of a raw value of optic density (OD). The antigen ratio is estimated by dividing the OD of the tested sample with the mean of eight negative samples plus three standard deviations (70).

\section{Questionnaire and Anthropometric Measurements}

A questionnaire was concurrently conducted while blood and stool samples were taken. The children were interviewed, and basic socio-demographic data (age, sex, residence) were recorded as well as specific questions about their living conditions and behaviors. Each child was assigned a unique ID code and removed their shoes so that we could measure their height (in $\mathrm{cm}$ ) and weight (in $\mathrm{kg}$ ).

\section{Statistical Analyses}

The data were identified and entered into a Microsoft Office Excel 2010 spreadsheet (Microsoft). Statistical analysis was performed using Stata SE 15.0 (StataCorp LLC, USA). We calculated the frequencies of categorical variables and made comparisons using Pearson's Chi-squared-test. Missing values were excluded for the comparisons. Continuous variables were described with means and standard deviations.

\section{RESULTS}

\section{Taeniasis Intestinal and Species Identification}

The coproparasitological analysis revealed an overall parasitism of $94.9 \%(639 / 673)$ by formol-ether concentration and a helminth parasitism percentage of $31.2 \%(211 / 675)$ by the KatoKatz technique. In two children [2/680 (0.3\%)], eggs of taeniid tapeworm were identified by both methods: Kato-Katz technique [2/675 (0.3\%)] and formol-ether concentration [2/673 (0.31\%)].

After parental consent, $2 \mathrm{~g}$ of Niclosamide and $1 \mathrm{ml}$ of Duphalac ${ }^{\circledR}$ oral solution (667 $\mathrm{mg} / \mathrm{ml}$ of lactulose) as a laxative were administered and the feces were collected. Two strobiles were recovered from both children and the morphologic study under a magnifying glass after injection with India ink revealed $<12$ uterine branches compatible with $T$. solium-proglottid demonstrating current tapeworm infection in both children.

Moreover, 144 samples were tested by coproantigen ELISA detection and $2 / 144(1.4 \%)$ samples had positive results. The positive samples obtained matched the positive samples obtained by Kato-Katz and formol-ether concentration techniques.

\section{Cysticercosis}

EITB analysis of a total of 572 sera samples revealed that $13.3 \%$ of the students $(76 / 572)$ presented reactivity to one or more of the specific glycoproteins of T. solium, of whom $46.0 \%(35 / 76)$ showed reactivity to three or more bands. A total of 7 of the 76 positive samples showed a single GP 50 band reaction, whereas in 9 of the positive samples, no GP 50 band was observed.

Exposure to $T$. solium was more frequent among girls than boys ( $p=0.014$, Table 1 ) and was especially prevalent in the Mucaca area of Nemba where $21.3 \%$ of the children had antiT. solium antibodies $(p<0.001)$. Children of all ages and in all classes had positive EITB results, and there was no obvious relationship with domestic animal rearing. The majority of the children came from homes that used a water pump as their main water source and had a latrine. Consumption of meat was significantly associated with $T$. solium exposure. The highest levels of exposure were among children that reported consuming meat once a month $(15.6 \%)$ or once a year (19.2\%); $13.1 \%$ of children who reported that they always washed their hands after going to the toilet had anti-T. solium antibodies, and this was not statistically different from children that reported they sometimes or never washed their hands (12.8 and $14.3 \%$, respectively).

Taenia solium antigens were found in 38\% (27/71) of children with anti-T. solium antibodies suggesting active infection. In addition, 14 of the 27 positive samples for antigen presented more than three bands by EIBT-test. None of the variables considered were associated with antigen presence among seropositive children (Table 1).

\section{DISCUSSION}

The present study demonstrates a substantial seroprevalence of cysticercosis in children of the Northern Province of Rwanda. The results suggest that more than of $13 \%$ of this group of children had been exposed to $T$ solium. In addition, among the reactive individuals, $46 \%$ had three or more reactive bands by EITB, which is highly suggestive of established cysticercosis infection. Furthermore, of the children with antibodies to cysticercosis, $38 \%$ were reactive to the detection of circulating antigen by ELISA, indicating the presence of active cases of neurocysticercosis, as previously demonstrated (65).

In 1982, Fain et al. said that T. solium was the most prevalent tapeworm in Rwanda (47). In previous studies in Rwanda, soiltransmitted helminths and schistosomiasis but no tapeworms were found. Since 1964, human taeniosis has not been detected in the country (51). However, analysis based on both microscopy or coproantigen detection of human fecal material cannot identify the species level, and the possibility of $T$. saginata presence cannot be ruled out. Other studies showed high parasitic burden strongly associated with drinking any kind of water (71-73).

The finding of eggs of Taenia spp. in two schoolchildren motivated us to deepen the search for associated human cysticercosis. Both children were positive based on microscopy (KK and FEC) and coproantigen detection (copro-Ag-ELISA).

In spite of false positives reported by coproantigen test (74), a high positivity value is highly predictive of a true positive (61). No cross-reactions were observed with other intestinal parasites (protozoa and helminths) present in the children. In this study, the microscopy and coproantigen detection assays had the same sensitivity as has been reported by other authors $(59,75)$. Our work showed some limitations because we were 
TABLE 1 | Results of the cysticercosis-specific tests conducted according to characteristics of the children.

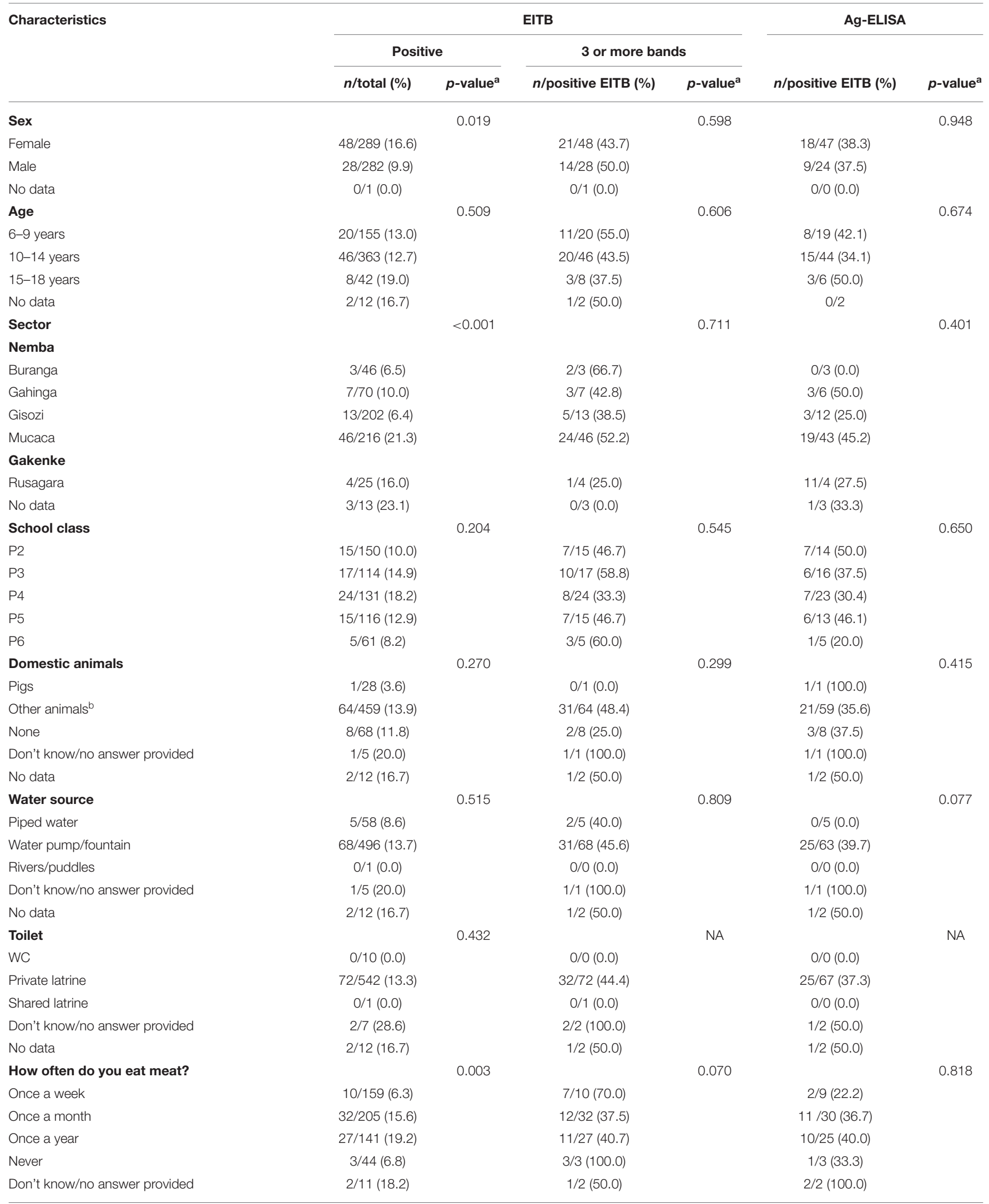


TABLE 1 | Continued

\begin{tabular}{|c|c|c|c|c|c|c|}
\hline \multirow[t]{3}{*}{ Characteristics } & \multicolumn{4}{|c|}{ EITB } & \multicolumn{2}{|l|}{ Ag-ELISA } \\
\hline & \multicolumn{2}{|c|}{ Positive } & \multicolumn{2}{|c|}{3 or more bands } & \multirow[b]{2}{*}{ n/positive EITB (\%) } & \multirow[b]{2}{*}{$p$-value } \\
\hline & n/total (\%) & $p$-value ${ }^{a}$ & n/positive EITB (\%) & $p$-value ${ }^{a}$ & & \\
\hline No data & 2/12 (16.7) & & $1 / 2(50.0)$ & & $1 / 2(50.0)$ & \\
\hline $\begin{array}{l}\text { How often do you wash your hands after going } \\
\text { to the toilet? }\end{array}$ & & 0.935 & & 0.367 & & 0.788 \\
\hline Always & 26/198 (13.1) & & 9/26 (34.6) & & $10 / 24(41.7)$ & \\
\hline Sometimes & $34 / 266$ (12.8) & & 18/34 (52.9) & & 12/34 (35.3) & \\
\hline Never & 13/91 (14.3) & & 6/13 (46.1) & & 3/10 (30.0) & \\
\hline Don't know/no answer provided & $1 / 5(20.0)$ & & 1/1 (100.0) & & 1/1 (100.0) & \\
\hline No data & 2/12 (16.7) & & $1 / 2(50.0)$ & & $1 / 2(50.0)$ & \\
\hline Total & 76/572 (13.3) & & $35 / 76(46.0)$ & & 27/71 (38.0) & \\
\hline
\end{tabular}

EITB, Anti-T. solium antibodies detection by enzyme-linked immunoelectrotransfer blot; Ag-ELISA, T. solium antigen detection by ELISA.

${ }^{a} p$-value from a Chi squared-test comparing answer categories, excluding missing or unknown values.

${ }^{b}$ Other animals: Cows, sheep, goats, rabbits, and chicken.

unable to carry out the coproantigen study on all samples, and the sample of children included came from a single school in the Gakenke district. However, participation was high, and with this study, we can confirm the presence of tapeworm carriers in this area. Additionally, we identified morphologically both adult specimens. Identification to species level in this country or bordering countries except in Tanzania has never been done (33).

To our knowledge, this is the first report of cysticercosis in schoolchildren in Rwanda. In cysticercosis surveys and immunodiagnostic tools applied on humans, serum samples are useful in estimating the prevalence and identifying of $T$. solium infection in the field. T. solium antigen in serum could predict the presence of viable brain parasites in patients with apparently calcified cysticercosis only (70). Antibody response in $T$. solium infection in field conditions was found to be a major contributor to the overestimation of the prevalence of cysticercosis in endemic areas $(4,76)$. The frequency found in these children was higher than prior literature reports in Butare, where $7 \%$ of autopsies were positive for cysticercosis (52). Furthermore, 21 and $21.8 \%$ of patients with epilepsy in Kigali and southern Rwanda, respectively, are positive for cysticercosis $(50,53,54)$. None of the previous studies were performed in children. In our study, it is important to highlight the early age of the population studied, since it is known that the prevalence of cysticercosis in a region increases with the age range studied. We can deduce that the seroprevalence in the adult population is likely to be higher than observed here.

The highest proportion of positive schoolchildren was observed in the same sector where tapeworm carriers were located. We observed small-scale swine production system in Mucaca (personal observation).

The financial losses due to human cysticercosis are very difficult to estimate but are certainly exceeded by the social impact of the disease, especially because of the particular perception of epilepsy in many African communities (14). Conditions like neurocysticercosis are better prevented than treated, because it has the potential to make a healthy person invalid. If the treatment is delayed, there can be irreversible brain damage.

In our study, no association was observed in frequency of hand washing after going to the toilet. However, in our study, close contact with pigs and use of latrines as possible risk factors for cysticercosis were not significantly associated with positive cases as observed in Tanzania (43). The absence of association may be explained by the level of widespread poverty in this area. Interestingly, meat consumption showed a significant association with cysticercosis exposure, but it was not a dose response. It is possible that people with limited resources cannot afford quality meat in these rural areas. Children who reported consuming meat once a month or once a year had higher exposure rates than those who reported consuming meat weekly, but it is possible that here we are seeing the impact of socio-economic status of the children rather than meat consumption itself. It has been shown that vegetarians and other people who do not eat pork can acquire cysticercosis through fecal-oral contamination with T. solium eggs from tapeworm carriers (77). Unhygienic sanitary conditions such as limited use or absence of latrines are prevalent in rural areas of Africa where pigs are raised $(14,78,79)$. Informally marketed foods, clandestine slaughtering of pigs, lack of trained and qualified meat inspectors, lack of detection and treatment of T. solium carriers, and consumption of undercooked or insufficiently cooked pork could be risk factors for taeniosis/cysticercosis among consumers $(22,26,35)$.

A nutritional survey and coproparasitological examination was being carried out at the Nemba 1 School, Gakenke district, Rwanda, when two samples harboring Taenia spp. eggs were found (unpublished results). It was impossible to obtain more samples or additional information that would allow the analysis of other risk factors or possible variables related to clinical manifestations of the infection. For the reasons stated, we decided to carry out the present study with the sole objective of investigating the presence of infection and transmission of 
T. solium in the community. The results obtained confirm the presence and transmission of T. solium and support the need to design more extensive future studies.

\section{CONCLUSIONS}

Overall, our study shows the presence and transmission of T. solium in Gakenke district. The high seroprevalence found in this area allows it to be considered hyperendemic for cysticercosis. To avoid neurological consequences, systematic investigations into porcine and human cysticercosis as well as health education and hygiene measures for T. solium control are needed. It is necessary to evaluate the clinical status of schoolchildren involved, carry out a study of contacts, and implement actions for the control and prevention of the disease.

\section{DATA AVAILABILITY STATEMENT}

All data analyzed during this study are included in this published article. The raw data or any further information supporting the conclusions of this article will be made available by the corresponding author on reasonable request.

\section{ETHICS STATEMENT}

The studies involving human participants were reviewed and approved by Experimental Research Commission on Ethics from University Miguel Hernández de Elche (Spain) (Ref: DF-MPA001-11). Written informed consent to participate in this study was provided by the participants' legal guardian/next of kin.

\section{REFERENCES}

1. Flisser A. Taeniasis and cysticercosis due to Taenia solium. Prog Clin Parasitol. (1994) 4:77-116.

2. García HH, Del Brutto OH. Imaging findings in neurocysticercosis. Acta Tropica. (2003) 87:71-8. doi: 10.1016/S0001-706X(03)00057-3

3. White AC. Neurocysticercosis: updates on epidemiology, pathogenesis, diagnosis, and management. Annu Rev Med. (2000) 51:187-206. doi: 10.1146/annurev.med.51.1.187

4. Garcia HH, Gonzalez AE, Gilman RH. Taenia solium Cysticercosis and its Impact in Neurological Disease. Clin Microbiol Rev. (2020) 33:e00085-19. doi: 10.1128/CMR.00085-19

5. Garcia HH, Del Brutto OH. Taenia solium cysticercosis. Infect Dis Clin North Am. (2000) 14:97-119. doi: 10.1016/S0891-5520(05)70220-8

6. Preux PM, Druet-Cabanac M. Epidemiology and aetiology of epilepsy in sub-Saharan Africa. Lancet Neurol. (2005) 4:2131. doi: 10.1016/S1474-4422(04)00963-9

7. WHO. First WHO Report on Neglected Tropical Diseases: Working to Overcome the Global Impact of Neglected Tropical Diseases. Geneva: World Health Organisation (2010). p. 1-184.

8. Sarti E, Rajshekhar V. Measures for the prevention and control of Taenia solium taeniosis and cysticercosis. Acta Tropica. (2003) 87:13743. doi: 10.1016/S0001-706X(03)00034-2

9. Nsadha Z, Thomas LF, Fèvre EM, Nasinyama G, Ojok L, Waiswa C. Prevalence of porcine cysticercosis in the Lake Kyoga Basin, Uganda. BMC Vet Res. (2014) 10:239. doi: 10.1186/s12917-014-0239-y

10. Wu HW, Ito $\mathrm{A}$, Ai L, Zhou XN, Acosta LP, Lee Willingham A. Cysticercosis/taeniasis endemicity in Southeast Asia: current

\section{AUTHOR CONTRIBUTIONS}

FB-L, HG, and JGE contributed to conception and design of the study. LA, LP, and FB-L supervised and coordinated the field study. LA, MI-G, and FB-L carried out the coproparasitological analysis in the field. CM-A and JGE coordinated the coproparasitological procedures. JB, YC, and EP coordinated and performed the serological and coproantigen tests. LA and LP organized the database-analyzed data, realized formal analysis, and interpretation of data. LA, LP, and HG wrote the first draft of the manuscript. FB-L critically reviewed it and contributed to draft design. All authors contributed to manuscript revision, read, and approved the submitted version.

\section{FUNDING}

This work was mainly supported by the Consejería de Bienestar Social, Generalitat Valenciana, Spain (Grant Number: 3055/2009); Centro de Cooperación al Desarrollo y Voluntariado (Universidad Miguel Hernández de Elche, Spain); and Programa de Becas Pre-doctorales Ciencias sin Fronteras (CAPES-Brasil). The funding bodies had no role in study design, data collection and analysis, decision to publish, or preparation of the manuscript.

\section{ACKNOWLEDGMENTS}

The authors thank the Laboratory of Nemba Hospital for their technical support and assistance in laboratory and field work duties and Jacinte for her translation. We thank all families for their collaboration and enthusiasm throughout the study.

status and control measures. Acta Trop. (2017) 165:12132. doi: 10.1016/j.actatropica.2016.01.013

11. Phiri IK, Ngowi H, Afonso S, Matenga E, Boa M, Mukaratirwa S, et al. The emergence of Taenia solium cysticercosis in Eastern and Southern Africa as a serious agricultural problem and public health risk. Acta Tropica. (2003) 87:13-23. doi: 10.1016/S0001-706X(03)00051-2

12. Willingham AL, Engels D. Control of Taenia solium cysticercosis/taeniosis. Adv Parasitol. (2006) 61:509-66. doi: 10.1016/S0065-308X(05)61012-3

13. O’Neal SE, Moyano LM, Ayvar V, Gonzalvez G, Diaz A, Rodriguez $\mathrm{S}$, et al. Geographic correlation between tapeworm carriers and heavily infected cysticercotic pigs. PLoS Negl Trop Dis. (2012) 12:1953. doi: 10.1371/journal.pntd.0001953

14. Zoli A, Shey-Njila O, Assana E, Nguekam JP, Dorny P, Brandt J, et al. Regional status, epidemiology and impact of Taenia solium cysticercosis in Western and Central Africa. Acta Tropica. (2003) 87:3542. doi: 10.1016/S0001-706X(03)00053-6

15. Braae UC, Saarnak CFL, Mukaratirwa S, Devleesschauwer B, Magnussen $\mathrm{P}$, Johansen MV. Taenia solium taeniosis/cysticercosis and the codistribution with schistosomiasis in Africa. Parasites Vectors. (2015) 8:323. doi: 10.1186/s13071-015-0938-7

16. Waiswa C, Fèvre EM, Nsadha Z, Sikasunge CS, Willingham AL. Porcine cysticercosis in Southeast Uganda: seroprevalence in Kamuli and Kaliro Districts. J Parasitol Res. (2009) 2009:1-5. doi: 10.1155/2009/375493

17. Kisakye J, Mal S. Short communication. Cysticcrcus ccllulosae in pigs slaughtered i and around Kampala city. Uganda J Agric Sci. (2002) 7:23-4. Available online at: https://www.ajol.info/index.php/ujas/article/view/128878

18. Kungu JM, Dione MM, Ejobi F, Harrison LJS, Poole EJ, Pezo D, et al. Sero-prevalence of Taenia spp. cysticercosis in rural and urban 
smallholder pig production settings in Uganda. Acta Trop. (2017) 165:1105. doi: 10.1016/j.actatropica.2016.01.016

19. Chartier C, Mutesi U, Ndakala NO. Les helminthes du porc domestique en Ituri, Haut-Zaïre. Ann Soc Belg Med Trop. (1990) 70:213-25.

20. Praet N, Kanobana K, Kabwe C, Maketa V, Lukanu P, Lutumba P, et al. Taenia solium cysticercosis in the democratic Republic of Congo: how does pork trade affect the transmission of the parasite?. PLoS Negl Trop Dis. (2010) 4:e817. doi: 10.1371/journal.pntd.0000817

21. Newell E, Vyungimana F, Geerts S, Van Kerckhoven I, Tsang VCW, Engels D. Prevalence of cysticercosis in epileptics and members of their families in Burundi. Trans R Soc Trop Med Hyg. (1997) 91:38991. doi: 10.1016/S0035-9203(97)90251-0

22. Boa ME, Mahundi EA, Kassuku AA, Willingham AL, Kyvsgaard NC. Epidemiological survey of swine cysticercosis using ante-mortem and postmortem examination tests in the southern highlands of Tanzania. Vet Parasitol. (2006) 139:249-55. doi: 10.1016/j.vetpar.2006.02.012

23. Mellau BL, Nonga HE, Karimuribo ED. Slaughter stock abattoir survey of carcasses and organ/offal condemnations in Arusha region, northern Tanzania. Trop Anim Health Prod. (2011) 43:857-64. doi: 10.1007/s11250-010-9773-1

24. Mkupasi EM, Ngowi HA, Nonga HE. Prevalence of extra-intestinal porcine helminth infections and assessment of sanitary conditions of pig slaughter slabs in Dar es Salaam city, Tanzania. Trop Anim Health Prod. (2011) 43:41723. doi: 10.1007/s11250-010-9708-x

25. Mkupasi EM, Ngowi HA, Sikasunge CS, Leifsson PS, Johansen MV. Efficacy of ivermectin and oxfendazole against Taenia solium cysticercosis and other parasitoses in naturally infected pigs. Acta Trop. (2013) 128:4853. doi: 10.1016/j.actatropica.2013.06.010

26. Komba EVG, Kimbi EC, Ngowi HA, Kimera SI, Mlangwa JE, Lekule FP, et al. Prevalence of porcine cysticercosis and associated risk factors in smallholder pig production systems in Mbeya region, southern highlands of Tanzania. Vet Parasitol. (2013) 198:284-91. doi: 10.1016/j.vetpar.2013.09.020

27. Ngowi HA, Kassuku AA, Maeda GEM, Boa ME, Carabin H, Willingham AL. Risk factors for the prevalence of porcine cysticercosis in Mbulu District, Tanzania. Vet Parasitol. (2004) 120:275-83. doi: 10.1016/j.vetpar.2004.01.015

28. Yohana C, Mwita C, Nkwengulila G. The Prevalence of Porcine Cysticercosis and Risk Factors for Taeniasis in Iringa Rural District. (2013). Available online at: http://repository.udsm.ac.tz:8080/xmlui/handle/123456789/1505 (accessed December 14, 2020).

29. Wilson RT, Swai E. A review of pig pathology in Tanzania. Trop Anim Health Prod. (2013) 45:1269-75. doi: 10.1007/s11250-013-0426-Z

30. Braae UC, Harrison W, Lekule F, Magnussen P, Johansen MV. Feedstuff and poor latrines may put pigs at risk of cysticercosis-A case-control study. Vet Parasitol. (2015) 214:187-91. doi: 10.1016/j.vetpar.2015.08.009

31. Kabatereine NB, Tukahebwa EM, Brooker S, Alderman $\mathrm{H}$, Hall A. Epidemiology of intestinal helminth infestations among schoolchildren in southern Uganda. East Afr Med J. (2001) 78:283-6. doi: 10.4314/eamj.v78i6.9019

32. Mwanjali G, Kihamia C, Kakoko DVC, Lekule F, Ngowi H, Johansen MV, et al. Prevalence and risk factors associated with human Taenia Solium infections in Mbozi District, Mbeya Region, Tanzania. PLoS Negl Trop Dis. (2013) 7:2102. doi: 10.1371/journal.pntd.0002102

33. Eom KS, Chai J-Y, Yong T-S, Min D-Y, Rim H-J, Kihamia C, et al. Morphologic and genetic identification of taenia tapeworms in Tanzania and DNA genotyping of Taenia solium. Korean J Parasitol. (2011) 49:399. doi: 10.3347/kjp.2011.49.4.399

34. Kanobana K, Praet N, Kabwe C, Dorny P, Lukanu P, Madinga J, et al. High prevalence of Taenia solium cysticerosis in a village community of Bas-Congo, Democratic Republic of Congo. Int J Parasitol. (2011) 41:10158. doi: 10.1016/j.ijpara.2011.06.004

35. Makita K, Fèvre EM, Waiswa C, Kaboyo W, Eisler MC, Welburn SC. Evidence-based identification of the most important livestock related zoonotic diseases in Kampala, Uganda. J Vet Med Sci. (2011) 73:49. doi: 10.1292/jvms.11-0049

36. Ngugi AK, Bottomley C, Kleinschmidt I, Wagner RG, Kakooza-Mwesige A, Ae-Ngibise K, et al. Prevalence of active convulsive epilepsy in sub-Saharan Africa and associated risk factors: cross-sectional and case-control studies. Lancet Neurol. (2013) 12:253-63. doi: 10.1016/S1474-4422(13)70003-6
37. Nzisabira L, Nsengiyumva G, Bouteille B, Ndayiragije A, Niyongabo T, Bigirimana V, et al. Cysticercosis in the province of Kayanza (Burundi). Bull Soc Pathol Exot. (1992) 85:374-7.

38. Nsengiyumva G, Druet-Cabanac M, Ramanankandrasana B, Bouteille B, Nsizabira L, Preux P-MM. Cysticercosis as a major risk factor for epilepsy in Burundi, East Africa. Epilepsia. (2003) 44:950-5. doi: 10.1046/j.1528-1157.2003.55302.x

39. Prado-Jean A, Kanobana K, Druet-Cabanac M, Nsengyiumva G, Dorny P, Preux PM, et al. Combined use of an antigen and antibody detection enzyme-linked immunosorbent assay for cysticercosis as tools in an epidemiological study of epilepsy in Burundi. Trop Med Int Heal. (2007) 12:895-901. doi: 10.1111/j.1365-3156.2007.01860.x

40. Diagana M, Nsengiyumva G, Tuillas M, Druet-Cabanac M, Bouteille B, Preux PM, et al. Électroencéphalogrammes réalisés chez 250 patients épileptiques dans une zone d'endémie cysticerquienne au Burundi. Neurophysiol Clin. (2005) 35:1-10. doi: 10.1016/j.neucli.2004.12.002

41. Mwang'onde BJ, Nkwengulila G, Chacha M. The risk factors for human cysticercosis in Mbulu District, Tanzania. Onderstepoort J Vet Res. (2014) 81:5. doi: $10.4102 /$ ojvr.v81i2.719

42. Winkler AS, Willingham AL, Sikasunge CS, Schmutzhard E. Epilepsy and neurocysticercosis in sub-Saharan Africa. Wiener Klinische Wochenschrift. (2009) 121:3-12. doi: 10.1007/s00508-009-1242-3

43. Blocher J, Schmutzhard E, Wilkins PP, Gupton PN, Schaffert M, Auer H, et al. A cross-sectional study of people with epilepsy and Neurocysticercosis in Tanzania: clinical characteristics and diagnostic approaches. PLoS Negl Trop Dis. (2011) 5:1185. doi: 10.1371/journal.pntd.0001185

44. Thienpont D, De Keyser J, Vandervelden M, Kageruka P. La Cysticercose ce're'brale du porc. Me'd Trop. (1959) 39:507-14.

45. Food and Agriculture Organization of the United Nations. Food Outlook: Biannual Report on Global Food Markets. Rome: ASHA Lead (2014). p. 18-9. Available online at: http://www.fao.org/3/i4136e/i4136e.pdf

46. Mushonga B, Habarugira G, Birori A, Kandiwa E, Samkange A, Bhebhe E. An epidemiological survey of the magnitude and local perceptions of porcine cysticercosis by two methods in Nyaruguru district, Rwanda. Vet Parasitol Reg Stud Rep. (2018) 14:18-24. doi: 10.1016/j.vprsr.2018.07.010

47. Fain A, Musafili I. Verminoses. In: Meheus A, Butera S, Eylenboceh W, Gatera G, Kivits M, Musafili I, editors. Sante et Maladies au Rwanda. Bruxelles: Administration Generaale de la Coopération au Développement (1982). p. 314-29.

48. Vanderick F, Fain A, Langi S, Vanbalen H. Deux noveaux cas de c'enurose humaine 'a Taenia brauni au Rwanda, avec une localisation orbitaire du c'enure. Ann Soc Belg Med Trop. (1920) 44:1077-9.

49. Gascón J, Corachan M, Ramiréz J. 1989 Gascón_Cysticercosis_5 cases Rwanda. Med Trop. (1989) 49:77-80.

50. Tuan J, Kailani L, Ngabitsinze P, Umuganwa S, Munyaneza F, Musoni E, et al. Disseminated cysticercosis in rwanda-case report of a patient presenting with difficulty with walking and skin nodules. Rwanda Med J. (2020) 77:30-33. Available online at: https://www.rwandamedicaljournal.org/uploads/1/2/2/1/ 122149944/cr.19.19.pdf

51. Scaglia M, Gatti S, Malfitano A, Strosselli M, Brusti R. Incidence of intestinal parasitosis among the Batwa and Hutu pygmy tribes of Rwanda. Bull Soc Pathol Exot Fil. (1983) 76:818-24.

52. Vanderick FX, Mboryingabo P. La cysticercose humaine au Rwanda [Humain cysticercosis in Rwanda]. Ann Soc Belg Med Trop. (1972) 52:153-5.

53. Tsang V, Wilson M. Taenia solium cysticercosis: an Unvider-recognized but serious public health problem. Parasitol Today. (1995) 11:1246. doi: 10.1016/0169-4758(95)80175-8

54. Rottbeck R, Nshimiyimana JF, Tugirimana P, Düll UE, Sattler J, Hategekimana JC, et al. High prevalence of cysticercosis in people with epilepsy in Southern Rwanda. PLoS Negl Trop Dis. (2013) 7:2558. doi: 10.1371 /journal.pntd.0002558

55. National Institute of Statistics of Rwanda. The Third Integrated Household Living Conditions Survey (EICV 3)-Main indicators Report. (2012). Available online at: https://www.statistics.gov.rw/publication/eicv-3-main-indicatorsreport

56. Goverment of Rwanda. Environmental and Management plan (ESMP). (2019). Available online at: https://gakenke.gov.rw/fileadmin/templates/document/ ESMP_GAKENKE.pdf 
57. Ash LR, Oriel TC, Savioli L. Bench aids for the diagnosis of intestinal parasites. World Health Org. (1996) 54:548. doi: 10.4269/ajtmh.1996.54.5.TM0540050548a

58. Knight WB, Hiatt RA, Cline BL, Ritchie LS. A modification of the formol ether concentration technique for increased sensitivity in detecting Schistosoma mansoni eggs. Am J Trop Med Hyg. (1976) 25:818-23. doi: 10.4269/ajtmh.1976.25.818

59. Allan JC, Craig PS, Avila G, Flisser A, Noval JG. Immunodiagnosis of taeniasis by coproantigen detection. Parasitology. (1990) 101:4737. doi: $10.1017 / S 0031182000060686$

60. Gonzalez AE, García HH, Gilman RH, Tsang VCW. Control of Taenia solium. Acta Tropica. (2003) 87:103-9. doi: 10.1016/S0001-706X(03)00025-1

61. Bustos JA, Rodriguez S, Jimenez JA, Moyano LM, Castillo Y, Ayvar V, et al. Detection of Taenia solium taeniasis coproantigen is an early indicator of treatment failure for taeniasis. Clin Vaccine Immunol. (2012) 19:5703. doi: 10.1128/CVI.05428-11

62. Tsang VCW, Brand JA, Boyer AE. An enzyme-linked immunoelectrotransfer blot assay and glycoprotein antigens for diagnosing human cysticercosis (Taenia solium). J Infect Dis. (1989) 159:50-9. doi: 10.1093/infdis/159.1.50

63. Gonzalez AE, Cama V, Gilman RH, Tsang VCW, Pilcher JB, Chavera A, et al. Prevalence and comparison of serologic assays, necropsy, and tongue examination for the diagnosis of porcine cysticercosis in Peru. Am J Trop Med Hyg. (1990) 43:194-9. doi: 10.4269/ajtmh.1990.43.194

64. Tsang VCW, Pilcher JA, Zhou W, Boyer AE, Kamango-Sollo EIP, Rhoads ML, et al. Efficacy of the immunoblot assay for cysticercosis in pigs and modulated expression of distinct IgM/ IgG activities to Taenia solium antigens in experimental infections. Vet Immunol Immunopathol. (1991) 29:69-78. doi: 10.1016/0165-2427(91)90053-F

65. Garcia HH, Harrison LJS, Parkhouse RME, Montenegro T, Martinez SM, Tsang VCW, et al. A specific antigen-detection ELISA for the diagnosis of human neurocysticercosis. Trans R Soc Trop Med Hyg. (1998) 92:4114. doi: 10.1016/S0035-9203(98)91069-0

66. Garcia HH. Serological diagnosis and follow-up of severe neurocysticercosis using HP10 antigen detection: commentary. Nat Clin Pract Neurol. (2007) 3:488-9. doi: $10.1038 /$ ncpneuro0563

67. Brandt JRA, Geerts S, Deken R De, Kumar V, Ceulemans F, Brijs L, et al. A monoclonal antibody-based ELISA for the detection of circulating excretorysecretory antigens in Taenia saginata cysticercosis. Int J Parasitol. (1992) 22:471-7. doi: 10.1016/0020-7519(92)90148-E

68. Van Kerckhoven I, Vansteenkiste W, Claes M, Geerts S, Brandt J. Improved detection of circulating antigen in cattle infected with Taenia saginata metacestodes. Vet Parasitol. (1998) 76:26974. doi: 10.1016/S0304-4017(97)00226-4

69. Dorny P, Phiri IK, Vercruysse J, Gabriel S, Willingham AL, Brandt J, et al. A Bayesian approach for estimating values for prevalence and diagnostic test characteristics of porcine cysticercosis. Int J Parasitol. (2004) 34:56976. doi: 10.1016/j.ijpara.2003.11.014

70. Zea-Vera A, Cordova EG, Rodriguez S, Gonzales I, Pretell EJ, Castillo Y, et al. Parasite Antigen in Serum Predicts the Presence of Viable Brain Parasites in
Patients With Apparently Calcified Cysticercosis Only. (2013). Available online at: https://academic.oup.com/cid/article-abstract/57/7/e154/337081 (accessed December 14, 2020).

71. Mupfasoni D, Karibushi B, Koukounari A, Ruberanziza E, Kaberuka T, Kramer MH, et al. Polyparasite helminth infections and their association to anaemia and undernutrition in Northern Rwanda. PLoS Negl Trop Dis. (2009) 3:517. doi: 10.1371/journal.pntd.0000517

72. Emile N, Bosco NJ, Karine B. Prevalence of intestinal parasitic infections and associated risk factors among Kigali Institute of Education students in Kigali, Rwanda. Trop Biomed. (2013) 30:718-26. Available online at: http://www. msptm.org/files/718_-_726_Bernard_Karine.pdf

73. Rujeni N, Morona D, Ruberanziza E, Mazigo HD. Schistosomiasis and soiltransmitted helminthiasis in Rwanda: an update on their epidemiology and control. Infect Dis Poverty. (2017) 6:8. doi: 10.1186/s40249-016-0212-z

74. Parkhouse RME, Rojas R G, Aguilar CM, Medina C, Ferrer E, Cortez Alcovedes MM. Diagnosis of taeniosis in rural venezuelan communities: preliminary characterization of a Taenia solium specific monoclonal (VP-1) coproantigen ELISA. Acta Trop. (2020) 207:105445. doi: 10.1016/j.actatropica.2020.105445

75. García HH, González AE, Evans CA, Gilman RH. Taenia solium Cysticercosis. (2003). Available online at: www.thelancet.com

76. Jayashi CM, Arroyo G, Lightowlers MW, García HH, Rodríguez S, Gonzalez AE. Seroprevalence and risk factors for Taenia solium cysticercosis in rural pigs of Northern Peru. PLoS Negl Trop Dis. (2012) 6:1733. doi: 10.1371/journal.pntd.0001733

77. Schantz PM, Moore AC, Muñoz JL, Hartman BJ, Schaefer JA, Aron AM, et al. Neurocysticercosis in an orthodox Jewish community in New York City. $N$ Engl J Med. (1992) 327:692-5. doi: 10.1056/NEJM199209033271004

78. Assana E, Lightowlers MW, Zoli AP, Geerts S. Taenia solium taeniosis/cysticercosis in Africa: risk factors, epidemiology and prospects for control using vaccination. Vet Parasitol. (2013) 195:14-23. doi: 10.1016/j.vetpar.2012.12.022

79. Gweba M, Faleke OO, Junaidu AU, Fabiyi JP, Fajinmi AO. Some risk factors for Taenia solium cysticercosis in semi-intensively raised pigs in Zuru, Nigeria. Vet Ital. (2010) 46:57-67. Available online at: https://www.izs.it/vet_italiana/ 2010/46_1/57.htm

Conflict of Interest: The authors declare that the research was conducted in the absence of any commercial or financial relationships that could be construed as a potential conflict of interest.

Copyright (๑ 2021 Acosta Soto, Parker, Irisarri-Gutiérrez, Bustos, Castillo, Perez, Muñoz-Antoli, Esteban, García and Bornay-Llinares. This is an open-access article distributed under the terms of the Creative Commons Attribution License (CC BY). The use, distribution or reproduction in other forums is permitted, provided the original author(s) and the copyright owner(s) are credited and that the original publication in this journal is cited, in accordance with accepted academic practice. No use, distribution or reproduction is permitted which does not comply with these terms. 